\title{
Quenching in a Non-Ideal Mechanical System and the Averaging Method
}

\author{
Márcio José Horta Dantas*, José Manoel Balthazar ${ }^{\dagger}$ and Jorge Luiz Palacios Felix** \\ * Faculdade de Matemática, UFU,38400-902 Uberlândia M.G. , BRAZIL \\ $\dagger$ Departamento de Estatística, Matemática Aplicada e Computação, UNESP,13506-900 RIO CLARO S.P., \\ BRAZIL \\ ** Departamento de Matemática, Universidade Federal do Pampa, 96413-170, BAGÉ, R.S., BRAZIL
}

\begin{abstract}
In this paper, for the first time, a quenching result in a non-ideal system is rigorously obtained. In order to do this a new mechanical hypothesis is assumed, it means that the moment of inertia of the rotating parts of the energy source is big. From this is possible to use the Averaging Method.
\end{abstract}

Keywords: Averaging Theorem, Non-Ideal System, Stability, Quenching PACS: $45.10 . \mathrm{Hj}$

\section{INTRODUCTION}

In the design of structures it is necessary to investigate the relevant dynamics in order to predict the structural response due to excitation. In the selection of rotating machines for applications in structures, often little thought is given to the effect that the structure has on the machine, i.e., the excitation is considered independent of the system response.

Mathematical models of real systems are usually idealized by prescribing the forcing term as a known function. In reality, for a great number of structures this is not the case, and such structures are called non-ideal. In general, it is possible that an energy source fixed to a structure may be affected by the structural response. Systems having dynamic coupling between structure and the energy source often exhibit peculiar behavior, especially systems with limited power supply. Non-ideal systems operating in the neighborhood of resonant frequencies are often more expensive and perform poorly as compared with ideal counterparts.A number of authors studied this class of mechanical systems. We can mention some of them Kononenko [8], Nayfeh and Mook [10] and Balthazar et al. [1], [2], [3].

In applications, for example Mechanical Engineering, a basic question is how to suppress, or at least, how to quench an undesirable vibration, see for example [11].

This work concerns with quenching in a class of nonideal mechanical systems. In [4] a numerical study is performed with same mechanical system that is investigated here. One of the motivations of [4] is the problem of vibration attenuation in a structure due to a nonlinear energy sink. Such kind of problem has been investigated by several authors such as Felix et all [5], Malatkar et all [9] and Jiang et all [7] in other mechanical systems.
In this paper, it is proved that a non ideal system exhibits a stable vibration and it is asked how to quench such oscillation. This is achieved by appending to the original system another one. Quenching of the original vibration has been achieved if there is a reduction of the $\left(x, x^{\prime}\right)$ amplitudes when comparing the initial system and the final one. It is worthy to note that all results are rigorously obtained by using the Averaging Theorem, see [6].

We organize this work as the following. In Section 2 the equations of motion of the non-ideal system under investigation are given. By assuming that angular momentum of the rotating parts of the motor is big, one has two scales, $\varepsilon, \varepsilon^{2}$, in the equations of motion of the system. These scales afford the correct use of Averaging Method in this problem. We believe that this approach is a step beyond of Kononenko's modelling, see [8]. In Section 3 it is shown that the last foregoing system has two hyperbolic periodic orbits, one of them is stable. In Section 4 a mass is attached, by springs, to the Main Body in the original system. It is proved that this new system has an asymptotically stable periodic orbit. And in Section 5 it is rigorously proved that the oscillations of the Main Body have been quenched in the new mechanical system. Finally, in Section 6 some comments are given.

\section{A NON-IDEAL SYSTEM}

In this section let us consider a mechanical system compound of a damped linear spring linked to a wall and to a principal body. On this principal body there is a $D C$ motor, with limited supply power, which rotates a small mass $m_{0}$. Both, the spring and the body-motor set, are at the same height from the ground and such system has 
two degrees of freedom. Such system is given in Figure 1.

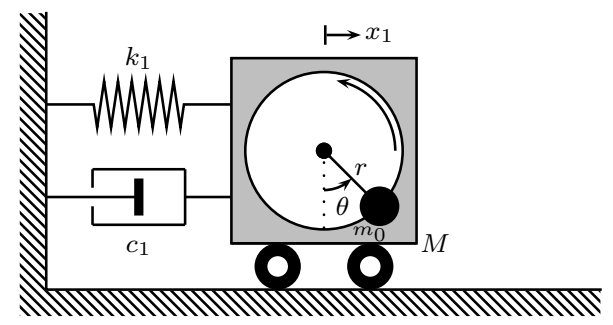

FIGURE 1. A Non Ideal System

In this system, $M_{0}$ denotes the mass of the fixed part of the mechanism such as its base. In Figure 1 this mass is indicated by the region in gray. The mass of the rotating parts of the motor is denoted by $M_{1}$ and its moment of inertia by $J$. Besides $M$, given by $M_{0}+M_{1}$, means the mass of the body-motor set. The constant $k_{1}$ is the stiffness of the spring. The resistance of the oscillatory motion is a linear force $c_{1} x_{1}^{\prime}$. Let $r$ be the distance between the mass $m_{0}$ and the axis of rotation of the $D C$ motor. It is assumed that $-\pi \leq \theta<\infty$.

The kinetic and potential are given respectively by

$$
\begin{aligned}
T= & \frac{M}{2}\left(x_{1}^{\prime}(t)\right)^{2} \\
& +\frac{m_{0}}{2}\left(\left(x_{1}^{\prime}(t)-r \theta^{\prime}(t) \cos \theta(t)\right)^{2}\right. \\
& \left.+\left(r \theta^{\prime}(t) \sin \theta(t)\right)^{2}\right)+\frac{J}{2} \theta^{\prime}(t)^{2}
\end{aligned}
$$

and

$$
V=\frac{k_{1}}{2}\left(x_{1}(t)\right)^{2}+g m_{0} r(1+\cos \theta(t)) .
$$

Let $L=T-V$ be the Lagrangian of this system then the equations of motion are given by

$$
\left\{\begin{array}{l}
\frac{d}{d t}\left(\frac{\partial L}{\partial x^{\prime}}\right)-\left(\frac{\partial L}{\partial x}\right)=-c_{1} x_{1}^{\prime}(t), \\
\frac{d}{d t}\left(\frac{\partial L}{\partial \theta^{\prime}}\right)-\left(\frac{\partial L}{\partial \theta}\right)=\Gamma_{0}\left(\theta^{\prime}(t)\right),
\end{array}\right.
$$

where function $\Gamma_{0}(\cdot)$ is the difference between the driving torque of the source of energy (motor) and the resistive torque applied to the rotor. Such function $\Gamma_{0}(\cdot)$ is obtained from experiments.

Let us define the following dimensionless variables and parameters

$$
\begin{aligned}
\tau & =\sqrt{\frac{k_{1}}{M+m_{0}}} t, \\
y_{1}(\tau) & =\frac{1}{r} x_{1}\left(\sqrt{\frac{M+m_{0}}{k_{1}}} \tau\right),
\end{aligned}
$$

$$
\begin{gathered}
\theta_{1}(\tau)=\theta\left(\sqrt{\frac{M+m_{0}}{k_{1}}} \tau\right), \\
\Gamma(z)=\frac{M+m_{0}}{k_{1} J} \Gamma_{0}\left(\sqrt{\frac{k_{1}}{M+m_{0}}} z\right) .
\end{gathered}
$$

By substituting the above equations in (3) one gets

$$
\left\{\begin{aligned}
y_{1}^{\prime \prime}(\tau)= & -y_{1}(\tau)-\frac{c_{1}}{\sqrt{k_{1}} \sqrt{M+m_{0}}} y_{1}^{\prime}(\tau) \\
& +\frac{m_{0}}{m_{0}+M}\left(\cos \left(\theta_{1}(\tau)\right)\left(\theta_{1}^{\prime \prime}(\tau)\right)\right. \\
& \left.-\sin \left(\theta_{1}(\tau)\right)\left(\theta_{1}^{\prime}(\tau)\right)^{2}\right), \\
\theta_{1}^{\prime \prime}(\tau)= & \frac{J}{J+m_{0} r^{2}} \Gamma\left(\theta_{1}^{\prime}(\tau)\right) \\
& +\frac{m_{0} g r\left(M+m_{0}\right)}{k_{1}\left(J+m_{0} r^{2}\right)} \sin \left(\theta_{1}(\tau)\right) \\
& +\frac{m_{0} r^{2}}{J+m_{0} r^{2}} \cos \left(\theta_{1}(\tau)\right) y_{1}^{\prime \prime}(\tau) .
\end{aligned}\right.
$$

It is assumed that $m_{0} \ll 1, c_{1} \ll 1, \Gamma(\cdot) \ll 1$ and $J \gg 1$. This last condition means that the moment of inertia of the rotating parts of the motor is big. In terms of adimensional parameters the following conditions are imposed:

$$
\begin{aligned}
\frac{c_{1}}{\sqrt{k_{1}} \sqrt{M+m_{0}}}=\varepsilon \lambda_{1}, \\
\frac{m_{0}}{m_{0}+M}=-\varepsilon q_{1}, \\
\frac{M_{0} r^{2}}{J}=\varepsilon q_{4},
\end{aligned}
$$

where $\lambda_{1}, q_{1}$ and $q_{4}$ are adimensional parameters. From (5), (6) and (7) one has

$$
\begin{gathered}
\frac{m_{0} r^{2}}{J+m_{0} r^{2}}=-\varepsilon^{2} q_{2}, \\
\frac{m_{0} g r\left(M+m_{0}\right)}{k_{1}\left(J+m_{0} r^{2}\right)}=\varepsilon^{2} q_{3},
\end{gathered}
$$

where $q_{2}$ and $q_{3}$ are dimensionless parameters.

In view of (8), (9), it will be assumed that

$$
\frac{J}{J+m_{0} r^{2}} \Gamma(\cdot)=\varepsilon^{2} \Gamma_{1}(\cdot),
$$

where $\Gamma_{1}(\cdot)$ is an adimensional function. Using (5), $(6),(8),(9)$ and (10) in (4), the following two scale system is obtained 


$$
\left\{\begin{aligned}
y_{1}^{\prime \prime}(\tau)= & -y_{1}(\tau)-\varepsilon \lambda_{1} y_{1}^{\prime}(\tau) \\
& -\varepsilon q_{1}\left(\cos \left(\theta_{1}(\tau)\right)\left(\theta_{1}^{\prime \prime}(\tau)\right)\right. \\
& \left.-\sin \left(\theta_{1}(\tau)\right)\left(\theta_{1}^{\prime}(\tau)\right)^{2}\right) \\
\theta_{1}^{\prime \prime}(\tau)= & \varepsilon^{2}\left(\Gamma_{1}\left(\theta_{1}^{\prime}(\tau)\right)\right. \\
& -q_{2} \cos \left(\theta_{1}(\tau)\right) y_{1}^{\prime \prime}(\tau) \\
& \left.+q_{3} \sin \left(\theta_{1}(\tau)\right)\right)
\end{aligned}\right.
$$

\section{STABLE AND UNSTABLE ORBITS}

From now on the following replacements in the notation will be done, $\tau \rightarrow t, y_{1} \rightarrow x_{1}, \theta_{1} \rightarrow \theta$ and $\Gamma_{1} \rightarrow \Gamma$. Of course it will be assumed that $t, x_{1}$ and $\Gamma$ are dimensionless. By rewritten (11) as a first order system, one gets

$$
\left\{\begin{aligned}
x_{1}^{\prime}(t)= & u(t), \\
u^{\prime}(t)= & \varepsilon\left(q_{1} p(t)^{2} \sin (\theta(t))\right. \\
& \left.-\lambda_{1} u(t)\right) \\
& -x_{1}(t)+\mathrm{O}\left(\varepsilon^{2}\right) \\
\theta^{\prime}(t)= & p(t) \\
p^{\prime}(t)= & \varepsilon^{2}\left(\Gamma(p(t))+q_{3} \sin (\theta(t))\right. \\
& \left.+q_{2} x_{1}(t) \cos (\theta(t))\right)+\mathrm{O}\left(\varepsilon^{3}\right) .
\end{aligned}\right.
$$

Following [8,pg.38], let us assume the frequency $p$ is close to natural frequency 1 . Thus

$$
p(t)=1+\varepsilon p_{1}(t) .
$$

By substituting (13) in (12) $)_{3}$ one gets $\theta^{\prime}(t)=1+\varepsilon p_{1}(t)$. Since $0<\varepsilon \leq 1$ then one can write $t$ in terms of $\theta$ and obtains the following reduced system

$$
\left\{\begin{aligned}
\bar{x}_{1}^{\prime}(\theta)= & \bar{u}(\theta)-\varepsilon \bar{p}_{1}(\theta)+\bar{u}(\theta) \\
& +\mathrm{O}\left(\varepsilon^{2}\right), \\
\bar{u}^{\prime}(\theta)= & -\bar{x}_{1}(\theta) \\
& +\varepsilon\left(\sin (\theta) q_{1}\right. \\
& \left.+\bar{p}_{1}(\theta) \bar{x}_{1}(\theta)-\lambda_{1} \bar{u}(\theta)\right) \\
& +\mathrm{O}\left(\varepsilon^{2}\right), \\
\bar{p}_{1}^{\prime}(\theta)= & \varepsilon\left(\sin (\theta) q_{3}\right. \\
& \left.+\bar{x}_{1}(\theta) \cos (\theta) q_{2}+\Gamma(1)\right) \\
& +\mathrm{O}\left(\varepsilon^{2}\right) .
\end{aligned}\right.
$$

Consider now the following change of variables

$$
\begin{aligned}
\bar{x}_{1}(\theta) & =\cos (\theta) x_{11}(\theta)+\sin (\theta) u_{1}(\theta) \\
\bar{u}(\theta) & =\cos (\theta) u_{1}(\theta)-\sin (\theta) x_{11}(\theta)
\end{aligned}
$$

By using (15) in (14) one obtains a non-autonomous system $2 \pi$ periodic in the $\theta$ variable. In this case the Averaging Theorem can be used. So, performing the average of this system on the interval $[0,2 \pi]$ one gets the following averaged system

$$
\left\{\begin{array}{l}
\widehat{x}_{11}^{\prime}(\theta)=\varepsilon \frac{-\lambda_{1} \widehat{x}_{11}-2 \widehat{p}_{1} \widehat{u}_{1}-q_{1}}{2}, \\
\widehat{u}^{\prime}(\theta)=\varepsilon \frac{2 p_{1} \widehat{x}_{11}-\lambda_{1} \widehat{u}_{1}}{2}, \\
\widehat{p}_{1}^{\prime}(\theta)=\varepsilon \frac{q_{2} \widehat{x}_{11}+2 \Gamma(1)}{2} .
\end{array}\right.
$$

The equilibrium solutions of (16) are given by

$$
\left\{\begin{array}{l}
\widehat{x}_{11}^{u n}=-\frac{2 \Gamma(1)}{q_{2}}, \\
\widehat{u}_{1}^{u n}=-\frac{\sqrt{2} \sqrt{\Gamma(1)} \sqrt{q_{1} q_{2}-2 \Gamma(1) \lambda_{1}}}{\sqrt{\lambda_{1}} q_{2}} \\
\widehat{p}_{1}^{u n}=\frac{\sqrt{\frac{\lambda_{1} q_{1} q_{2}}{\Gamma(1)}-2 \lambda_{1}^{2}}}{2^{\frac{3}{2}}}
\end{array}\right.
$$

and 


$$
\left\{\begin{array}{l}
\widehat{x}_{11}^{s}=-\frac{2 \Gamma(1)}{q_{2}}, \\
\widehat{u}_{1}^{s}=\frac{\sqrt{2} \sqrt{\Gamma(1)} \sqrt{q_{1} q_{2}-2 \Gamma(1) \lambda_{1}}}{\sqrt{\lambda_{1} q_{2}}} \\
\widehat{p}_{1}^{s}=-\frac{\sqrt{\frac{\lambda_{1} q_{1} q_{2}}{\Gamma(1)}-2 \lambda_{1}^{2}}}{2^{\frac{3}{2}}} .
\end{array}\right.
$$

Of course the following inequalities must be hold in order (17) and (18) could be valid.

$$
\begin{gathered}
\lambda_{1}>0, \\
\Gamma(1)>0, \\
q_{1} q_{2}-2 \Gamma(1) \lambda_{1}>0 .
\end{gathered}
$$

The eigenvalues of the jacobian of the vector field given by the right side of (16) calculated at the equilibrium point (17) are roots of the characteristic polinomial $P(\lambda)=\lambda^{3}+A \lambda^{2}+B \lambda+C$, where

$$
\begin{aligned}
& A=\lambda_{1}, \\
& B=-\frac{2^{\frac{5}{2}} \Gamma(1)^{\frac{3}{2}} \sqrt{q_{1} q_{2}-2 \Gamma(1) \lambda_{1}}-\lambda_{1}^{\frac{3}{2}} q_{1} q_{2}}{8 \Gamma(1) \sqrt{\lambda_{1}}}, \\
& C=-\frac{\sqrt{\Gamma(1)} \sqrt{\lambda_{1}} \sqrt{q_{1} q_{2}-2 \Gamma(1) \lambda_{1}}}{\sqrt{2}}, \\
& A B-C=\frac{\lambda_{1}^{2} q_{1} q_{2}}{8 \Gamma(1)} .
\end{aligned}
$$

From $(20)_{3}$ one concludes that $C$ is negative thus it follows from Hurwitz Criterium that $P(\lambda)$ has a root which real part is positive. Then one concludes from Averaging Theorem that (14) has an unstable hyperbolic periodic orbit.

In the case of the equilibrium point given by (18) one gets

$$
\begin{aligned}
& A=\lambda_{1}, \\
& B=\frac{2^{\frac{5}{2}} \Gamma(1)^{\frac{3}{2}} \sqrt{q_{1} q_{2}-2 \Gamma(1) \lambda_{1}}+\lambda_{1}^{\frac{3}{2}} q_{1} q_{2}}{8 \Gamma(1) \sqrt{\lambda_{1}}}, \\
& C=\frac{\sqrt{\Gamma(1)} \sqrt{\lambda_{1}} \sqrt{q_{1} q_{2}-2 \Gamma(1) \lambda_{1}}}{\sqrt{2}}, \\
& A B-C=\frac{\lambda_{1}^{2} q_{1} q_{2}}{8 \Gamma(1)} .
\end{aligned}
$$

\begin{tabular}{|c|c|c|c|}
\hline \multicolumn{2}{|c|}{ Parameters } & \multicolumn{2}{|c|}{ Initial Conditions } \\
\hline$\varepsilon$ & 0.01 & Stable Case & Unstable Case \\
\hline$q_{1}$ & -2 & $x_{1}(0)=0.66$ & $x_{1}(0)=0.66$ \\
\hline$q_{2}$ & -3 & $u(0)=-1.49$ & $u(0)=1.49$ \\
\hline$q_{3}$ & 0.1 & $p_{1}(0)=-0.55$ & $p_{1}(0)=0.55$ \\
\hline$\gamma_{1}$ & 0.5 & & \\
\hline$a$ & 6 & & \\
\hline$b$ & 5 & & \\
\hline
\end{tabular}

So all roots of $P(\lambda)$ have negative real parts, thus it follows from Averaging Theorem that (14) has an asymptotically stable hyperbolic periodic orbit.

As it was assumed in [4], let us take $\Gamma\left(\varphi^{\prime}\right)=a-b \varphi^{\prime}$, where $a$ and $b$ are adimensional parameters. For physical motivation of this choice see [4, pg.2]. Now, using the parameters given at the following table, the equations (18), (17) and the Averaging Theorem, one can obtain the initial conditions for the cases stable and unstable of (14). The final results are given in the next table.

Using this data, one gets the following graphics.

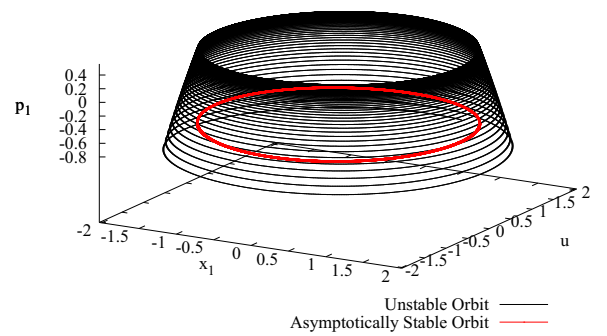

FIGURE 2. The Stable and Unstable Orbits

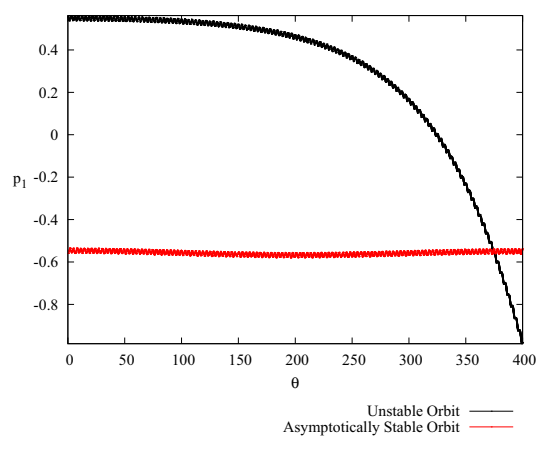

FIGURE 3. $\quad \theta \times p_{1}$ Graphic

\section{ANOTHER NON IDEAL SYSTEM}

Although the existence of an asymptotically stable periodic orbit obtained in Section 3, it should be interesting to suppress or at least to quench this orbit. The way we 
chose is to append a mass to the principal one, given at Figure 1 in a way given in the following figure.

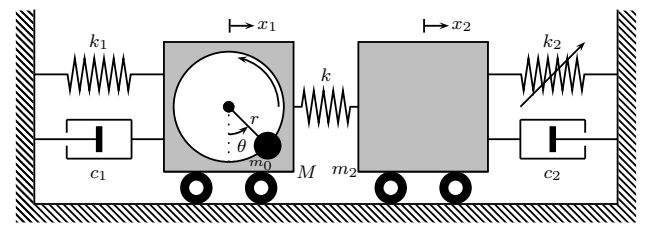

FIGURE 4. A Non-Ideal System with Three Degree of Freedom

The equations of motion of the mechanical system given in Figure 4, in its dimensionless form and after the introduction of a small parameter $\varepsilon$ as in Section 3, are given by

$$
\left\{\begin{aligned}
x_{1}^{\prime \prime}(t)= & -x_{1}(t) \\
& +\varepsilon_{1}\left(x_{2}(t)-x_{1}(t)\right)-\varepsilon \lambda_{1} x_{1}^{\prime}(t) \\
& +\varepsilon q_{1}\left(\sin (\theta(t))\left(\theta^{\prime}(t)\right)^{2}\right. \\
& \left.-\cos (\theta(t)) \theta^{\prime \prime}(t)\right) \\
x_{2}^{\prime \prime}(t)= & \varepsilon_{2}\left(x_{1}(t)-x_{2}(t)\right)-\varepsilon \lambda_{2} x_{2}^{\prime}(t) \\
& -\varepsilon \gamma x_{2}(t)^{3}, \\
\theta^{\prime \prime}(t)= & \varepsilon^{2}\left(\Gamma\left(\theta^{\prime}(t)\right)\right. \\
& -q_{2} \cos (\theta(t))\left(x_{1}^{\prime \prime}(t)\right) \\
& \left.+q_{3} \sin (\theta(t))\right) .
\end{aligned}\right.
$$

Of course the parameters in (22) depend on the physical constants $k_{1}, k_{2}, k, M, m_{0}, m_{2}, r$ as well as of the moment of inertia of rotating parts and acceleration of gravity. As in the Section 2, the function $\Gamma$ is the difference between the driving torque of the source energy, in this case a motor, and the resistive torque applied to the rotor. Again this function is given in its dimensionless form. We will write (22) as a pair of perturbed harmonic oscillators coupled with $(22)_{3}$.

Let $r_{1}, r_{2}$ be positive parameters such that $r_{2}<1<r_{1}$ and

$$
\begin{aligned}
& \varepsilon_{1}=\left(r_{1}^{2}-1\right)\left(1-r_{2}^{2}\right) \\
& \varepsilon_{2}=r_{1}^{2} r_{2}^{2} .
\end{aligned}
$$

By writing (22) as a first order system, using (23) and the following change of variables

$$
\left(\begin{array}{c}
x_{1}(t) \\
u(t) \\
x_{2}(t) \\
v(t) \\
\theta(t) \\
p(t)
\end{array}\right)=\left(\begin{array}{c}
x_{21}(t)+x_{11}(t) \\
r_{1} v_{1}(t)+r_{2} u_{1}(t) \\
\frac{r_{2}^{2} x_{21}(t)}{r_{2}^{2}-1}+\frac{r_{1}^{2} x_{11}(t)}{r_{1}^{2}-1} \\
\frac{r_{1} r_{2}^{2} v_{1}(t)}{r_{2}^{2}-1}+\frac{r_{1}^{2} r_{2} u_{1}(t)}{r_{1}^{2}-1} \\
\theta(t) \\
p(t)
\end{array}\right)
$$

where $u(t)=x_{1}^{\prime}(t), v(t)=x_{2}^{\prime}(t)$ and $p(t)=\theta^{\prime}(t)$, one obtains the following system

$$
\begin{aligned}
x_{11}^{\prime}(t)= & u_{1}(t), \\
u_{1}^{\prime}(t)= & -r_{2}^{2} x_{11}(t) \\
& +\varepsilon_{c_{3}}\left(c _ { 1 } \left(q_{1} p(t)^{2} \sin (\theta(t))\right.\right. \\
& \left.-\lambda_{1}\left(v_{1}(t)+u_{1}(t)\right)\right) \\
& +c_{2}\left(-\gamma\left(c_{7} x_{21}(t)+c_{8} x_{11}(t)\right)^{3}\right. \\
& \left.\left.-\lambda_{2}\left(c_{7} v_{1}(t)+c_{8} u_{1}(t)\right)\right)\right) \\
& +O\left(\varepsilon^{2}\right), \\
x_{21}^{\prime}(t)= & v_{1}(t), \\
v_{1}^{\prime}(t)= & -r_{1}^{2} x_{21}(t) \\
& +\varepsilon_{1}^{r_{3}}\left(c _ { 4 } \left(q_{1} p(t)^{2} \sin (\theta(t))\right.\right. \\
& \left.-\lambda_{1}\left(v_{1}(t)+u_{1}(t)\right)\right) \\
& +c_{5}\left(-\gamma\left(c_{7} x_{21}(t)+c_{8} x_{11}(t)\right)^{3}\right. \\
& \left.\left.-\lambda_{2}\left(c_{7} v_{1}(t)+c_{8} u_{1}(t)\right)\right)\right) \\
& +O\left(\varepsilon^{2}\right), \\
\theta^{\prime}(t)= & p(t), \\
p^{\prime}(t)=\quad & \varepsilon^{2}\left(\Gamma(p(t))+q_{3} \sin (\theta(t))\right. \\
& +\left(c_{9}\left(x_{21}(t)+x_{11}(t)\right)\right. \\
& \left.-c_{6} q_{2}\left(c_{7} x_{21}(t)+c_{8} x_{11}(t)\right)\right) \\
& \cos (t))), \\
= & \\
= &
\end{aligned}
$$

where $c_{1}, \ldots, c_{9}$ are adequate rational functions of $r_{1}, r_{2}$. Assuming now the ansatz

$$
p(t)=\omega_{0}+\varepsilon p_{1}(t),
$$


where $\omega_{0}$ is parameter to be chosen later. By following analogous steps to those ones performed in Section 3, see (13), one can obtain a $\theta$ dependent reduced system from (25) This new system has five equations and its unknown funtions are given by $\bar{x}_{11}(\theta), \bar{u}_{1}(\theta), \bar{x}_{21}(\theta)$, $\bar{v}_{1}(\theta)$ and $\bar{p}_{1}(\theta)$. In this new system is applied the following change of variables

$$
\left(\begin{array}{c}
\bar{x}_{11} \\
\bar{u}_{1} \\
\bar{x}_{21} \\
\bar{v}_{1}
\end{array}\right)=\left(\begin{array}{c}
\cos \left(\frac{\theta r_{2}}{\omega_{0}}\right) z_{11}+\frac{\sin \left(\frac{\theta r_{2}}{\omega_{0}}\right) z_{1}}{r_{2}} \\
\cos \left(\frac{\theta r_{2}}{\omega_{0}}\right) z_{1}-r_{2} \sin \left(\frac{\theta r_{2}}{\omega_{0}}\right) z_{11} \\
\cos \left(\frac{\theta r_{1}}{\omega_{0}}\right) z_{21}+\frac{\sin \left(\frac{\theta r_{1}}{\omega_{0}}\right) z_{2}}{r_{1}} \\
\cos \left(\frac{\theta r_{1}}{\omega_{0}}\right) z_{2}-r_{1} \sin \left(\frac{\theta r_{1}}{\omega_{0}}\right) z_{21}
\end{array}\right) .
$$

By using (27) in the $\theta$ dependent system described earlier one gets

$$
\left(\begin{array}{c}
z_{11}^{\prime} \\
z_{1}^{\prime} \\
z_{21}^{\prime} \\
z_{2}^{\prime} \\
\bar{p}_{1}^{\prime}
\end{array}\right)=\varepsilon\left(\begin{array}{l}
G_{1}\left(z_{11}, z_{1}, z_{21}, z_{2}, \bar{p}_{1}, \theta\right) \\
G_{2}\left(z_{11}, z_{1}, z_{21}, z_{2}, \bar{p}_{1}, \theta\right) \\
G_{3}\left(z_{11}, z_{1}, z_{21}, z_{2}, \bar{p}_{1}, \theta\right) \\
G_{4}\left(z_{11}, z_{1}, z_{21}, z_{2}, \bar{p}_{1}, \theta\right) \\
G_{5}\left(z_{11}, z_{1}, z_{21}, z_{2}, \bar{p}_{1}, \theta\right)
\end{array}\right)+O\left(\varepsilon^{2}\right)
$$

where $G_{j}, j=1, \ldots, 5$ are $C^{\infty}$ functions . Their algebraic expressions are very complicated and we will not give them here. Now, assuming that

$$
\omega_{0}=r_{1}
$$

let us adopt the following resonance condition

$$
r_{1}=\frac{3}{2} r_{2}
$$

Substituting (29),(30) in (28) one obtains another $\theta$ dependent system with period equal to $6 \pi$. Computing the averaged system, one has

$$
\left(\begin{array}{c}
\widehat{z}_{11}^{\prime} \\
\widehat{z}_{1}^{\prime} \\
\widehat{z}_{21}^{\prime} \\
\widehat{z}_{2}^{\prime} \\
\widehat{p}_{1}^{\prime}
\end{array}\right)=\varepsilon\left(\begin{array}{l}
F_{1}\left(\widehat{z}_{11}, \widehat{z}_{1}, \widehat{z}_{21}, \widehat{z}_{2}, \widehat{p}_{1}\right) \\
F_{2}\left(\widehat{z}_{11}, \widehat{z}_{1}, \widehat{z}_{21}, \widehat{z}_{2}, \widehat{p}_{1}\right) \\
F_{3}\left(\widehat{z}_{11}, \widehat{z}_{1}, \widehat{z}_{21}, \widehat{z}_{2}, \widehat{p}_{1}\right) \\
F_{4}\left(\widehat{z}_{11}, \widehat{z}_{1}, \widehat{z}_{21}, \widehat{z}_{2}, \widehat{p}_{1}\right) \\
F_{5}\left(\widehat{z}_{11}, \widehat{z}_{1}, \widehat{z}_{21}, \widehat{z}_{2}, \widehat{p}_{1}\right)
\end{array}\right)
$$

where

$$
F_{1}=\frac{\left(\begin{array}{l}
\left(18 \pi c_{2} c_{7}^{2} c_{8} \gamma r_{2}{ }^{2} \widehat{z}_{1} \widehat{z}_{21}^{2}\right. \\
+8 \pi c_{2} c_{7}{ }^{2} c_{8} \gamma \widehat{z}_{1} \widehat{z}_{2}^{2} \\
+9 \pi c_{2} c_{8}{ }^{3} \gamma r_{2}{ }^{2} \widehat{z}_{1} \widehat{z}_{11}^{2} \\
+\left(-12 \pi \lambda_{2} c_{2} c_{8}-12 \pi \lambda_{1} c_{1}\right) r_{2}{ }^{4} \widehat{z}_{11} \\
\left.+9 \pi c_{2} c_{8}{ }^{3} \gamma \widehat{z}_{1}^{3}-16 \pi c_{3} \widehat{p}_{1} r_{2}{ }^{2} \widehat{z}_{1}\right)
\end{array}\right)}{36 \pi c_{3} r_{2}{ }^{4}}
$$

$$
\begin{aligned}
& F_{2}=-\frac{\left(\begin{array}{l}
\left(18 \pi c_{2} c_{7}^{2} c_{8} \gamma r_{2}{ }^{2} \widehat{z}_{11} \widehat{z}_{21}^{2}\right. \\
+8 \pi c_{2} c_{7}^{2} c_{8} \gamma \widehat{z}_{11} \widehat{z}_{2}^{2} \\
+9 \pi c_{2} c_{8}^{3} \gamma r_{2}{ }^{2} \widehat{z}_{11}^{3} \\
+\left(9 \pi c_{2} c_{8}^{3} \gamma \widehat{z}_{1}^{2}-16 \pi c_{3} \widehat{p}_{1} r_{2}^{2}\right) \widehat{z}_{11} \\
\left.+\left(12 \pi \lambda_{2} c_{2} c_{8}+12 \pi \lambda_{1} c_{1}\right) r_{2} \widehat{z}_{1}\right)
\end{array}\right)}{36 \pi c_{3} r_{2}{ }^{2}} \\
& F_{3}=\frac{\left(\begin{array}{l}
\left(18 \pi c_{5} c_{7}{ }^{3} \gamma r_{2}{ }^{2} \widehat{z}_{2} \widehat{z}_{21}^{2}\right. \\
+\left(-54 \pi \lambda_{2} c_{5} c_{7}-54 \pi \lambda_{1} c_{4}\right) r_{2}{ }^{4} \widehat{z}_{21} \\
+8 \pi c_{5} c_{7}{ }^{3} \gamma \widehat{z}_{2}^{3} \\
+\left(36 \pi c_{5} c_{7} c_{8}{ }^{2} \gamma r_{2}{ }^{2} \widehat{z}_{11}^{2}\right. \\
\left.+36 \pi c_{5} c_{7} c_{8}{ }^{2} \gamma \widehat{z}_{1}^{2}-48 \pi c_{3} \widehat{p}_{1} r_{2}{ }^{2}\right) \widehat{z}_{2} \\
\left.-81 \pi c_{4} q_{1} r_{2}{ }^{5}\right)
\end{array}\right)}{108 \pi c_{3} r_{2}{ }^{4}} \\
& F_{4}=-\frac{\left(\begin{array}{l}
\left(9 \pi c_{5} c_{7}{ }^{3} \gamma r_{2}{ }^{2} \widehat{z}_{21}^{3}\right. \\
+\left(4 \pi c_{5} c_{7}{ }^{3} \gamma \widehat{z}_{2}^{2}\right. \\
+18 \pi c_{5} c_{7} c_{8}{ }^{2} \gamma r_{2}{ }^{2} \widehat{z}_{11}^{2} \\
+18 \pi c_{5} c_{7} c_{8}{ }^{2} \gamma \widehat{z}_{1}^{2} \\
\left.-24 \pi c_{3} \widehat{p}_{1} r_{2}{ }^{2}\right) \widehat{z}_{21} \\
\left.+\left(12 \pi \lambda_{2} c_{5} c_{7}+12 \pi \lambda_{1} c_{4}\right) r_{2}{ }^{2} \widehat{z}_{2}\right)
\end{array}\right)}{24 \pi c_{3} r_{2}{ }^{2}} \\
& F_{5}=\frac{\left(18 \pi c_{9}-18 \pi c_{6} c_{7} q_{2}\right) r_{2} \widehat{z}_{21}+36 \pi r_{2} \Gamma\left(\frac{3 r_{2}}{2}\right)}{54 \pi r_{2}{ }^{2}}
\end{aligned}
$$

An equilibrium point of (31) is given by

$$
\begin{aligned}
& \widehat{z}_{11}^{s}=0, \widehat{z}_{1}^{s}=0, \widehat{z}_{21}^{s}=-\frac{8 \Gamma\left(\frac{3 r_{2}}{2}\right)}{9 q_{2} r_{2}^{2}}, \\
& \widehat{z}_{2}^{s}=\frac{1}{3 q_{2} r_{2}} \sqrt{\frac{R_{1}}{R_{2}} \Gamma\left(\frac{3 r_{2}}{2}\right)}, \\
& \widehat{p}_{1}^{s}=-\frac{\left(\begin{array}{c}
24 \gamma q_{1} r_{2}^{2}\left(9 r_{2}^{2}-4\right) \sqrt{R_{2}} \Gamma\left(\frac{3 r_{2}}{2}\right)^{2} \\
+\sqrt{R_{1}} q_{2}\left(r_{2}^{2}-1\right) R_{2}^{2} \sqrt{\Gamma\left(\frac{3 r_{2}}{2}\right)}
\end{array}\right)}{40 q_{2}\left(r_{2}^{2}-1\right) R_{2}^{\frac{3}{2}} \Gamma\left(\frac{3 r_{2}}{2}\right)} .
\end{aligned}
$$

Of course the parameters $c_{i}$ were substituted by the original ones. It is assumed that the following inequalities hold:

$$
\begin{array}{r}
\Gamma\left(\frac{3 r_{2}}{2}\right)>0, \\
R_{1}=243 q_{1} q_{2}\left(1-r_{2}^{2}\right) r_{2}{ }^{3}-16 R_{2} \Gamma\left(\frac{3 r_{2}}{2}\right)>0, \\
R_{2}=\left(9 \lambda_{2}-9 \lambda_{1}\right) r_{2}^{2}-4 \lambda_{2}+9 \lambda_{1}>0 .
\end{array}
$$

Hence, computing the jacobian of (31) at the equilibrium point (32) one gets the matrix $M_{1} \oplus M_{2}$ where

$$
M_{1}=\left(\begin{array}{cc}
A & B \\
-r_{2}^{2} B & A
\end{array}\right),
$$




$$
A=\frac{\left(9 \lambda_{2}-9 \lambda_{1}\right) r_{2}^{2}-9 \lambda_{2}+4 \lambda_{1}}{15 r_{2}}
$$

and

$$
B=\frac{\left(\begin{array}{c}
12 \gamma q_{1} r_{2}^{2}\left(99 r_{2}^{2}-89\right) \Gamma\left(\frac{3 r_{2}}{2}\right)^{2} \\
+\sqrt{R_{1}} q_{2}\left(r_{2}^{2}-1\right) R_{2}^{\frac{3}{2}} \sqrt{\Gamma\left(\frac{3 r_{2}}{2}\right)}
\end{array}\right)}{90 q_{2} r_{2}^{2}\left(r_{2}^{2}-1\right) R_{2} \Gamma\left(\frac{3 r_{2}}{2}\right)}
$$

Besides $M_{2}$ is given by

$$
M_{2}=\left(\begin{array}{lll}
M_{211} & M_{212} & M_{213} \\
M_{221} & M_{222} & M_{223} \\
M_{231} & M_{232} & M_{233}
\end{array}\right)
$$

where

$$
\begin{aligned}
& M_{211}=-\frac{\left(\begin{array}{l}
\sqrt{R_{1}}\left(144 \gamma r_{2}^{2}-64 \gamma\right) \Gamma\left(\frac{3 r_{2}}{2}\right)^{\frac{3}{2}} \\
+81 R_{2}^{\frac{3}{2}} q_{2}^{2}\left(r_{2}^{2}-1\right)^{2} r_{2}
\end{array}\right)}{1215 \sqrt{R_{2}} q_{2}^{2}\left(r_{2}^{2}-1\right)^{2} r_{2}^{2}}, \\
& M_{221}=-\frac{\left(\begin{array}{l}
\left(2304 \gamma r_{2}^{2}-1024 \gamma\right) \Gamma\left(\frac{3 r_{2}}{2}\right)^{3} \\
+81 \sqrt{R_{1} R_{2}} q_{2}^{2}\left(r_{2}^{2}-1\right)^{2} r_{2} \sqrt{\Gamma\left(\frac{3 r_{2}}{2}\right)}
\end{array}\right)}{3240 q_{2}^{2}\left(r_{2}^{2}-1\right)^{2} r_{2} \Gamma\left(\frac{3 r_{2}}{2}\right)}, \\
& M_{231}=\frac{3 q_{2} r_{2}}{4}, \\
& M_{212}=\frac{\left(\begin{array}{l}
-\left(9 r_{2}^{2}-4\right)\left(256 R_{2} \gamma \Gamma\left(\frac{3 r_{2}}{2}\right)^{3}\right. \\
\left.+3888 \gamma q_{1} q_{2}\left(r_{2}^{2}-1\right) r_{2}^{3} \Gamma\left(\frac{3 r_{2}}{2}\right)^{2}\right) \\
+81 \sqrt{R_{1}} R_{2}^{\frac{3}{2}} q_{2}^{2}\left(r_{2}^{2}-1\right)^{2} r_{2} \sqrt{\Gamma\left(\frac{3 r_{2}}{2}\right)}
\end{array}\right)}{7290 R_{2} q_{2}^{2}\left(r_{2}^{2}-1\right)^{2} r_{2}{ }^{3} \Gamma\left(\frac{3 r_{2}}{2}\right)}, \\
& M_{222}=\frac{\left(\begin{array}{l}
\sqrt{R_{1}}\left(144 \gamma r_{2}{ }^{2}-64 \gamma\right) \Gamma\left(\frac{3 r_{2}}{2}\right)^{\frac{3}{2}} \\
-81 R_{2}^{\frac{3}{2}} q_{2}^{2}\left(r_{2}^{2}-1\right)^{2} r_{2}
\end{array}\right)}{1215 \sqrt{R_{2}} q_{2}^{2}\left(r_{2}^{2}-1\right)^{2} r_{2}{ }^{2}}, \\
& M_{232}=0, \quad M_{213}=-\frac{4 \sqrt{R_{1}} \sqrt{\Gamma\left(\frac{3 r_{2}}{2}\right)}}{27 \sqrt{R_{2}} q_{2} r_{2}^{3}}, \\
& M_{223}=-\frac{8 \Gamma\left(\frac{3 r_{2}}{2}\right)}{9 q_{2} r_{2}{ }^{2}}, \quad M_{233}=0 .
\end{aligned}
$$

Since we are interested in stable periodic orbits, we have to find out sufficient conditions on the parameters such that all eigenvalues of the jacobian $M_{1} \oplus M_{2}$ have negative real parts. For $M_{1}$, in view of (36) and since $\operatorname{det}\left(M_{1}\right)=A^{2}+B^{2} r_{2}^{2}>0$ the following inequality

$$
\left(9 \lambda_{2}-9 \lambda_{1}\right) r_{2}^{2}-9 \lambda_{2}+4 \lambda_{1}<0
$$

is enough.

The characteristic polynomial of (37) is given by $p(s)=s^{3}+A_{1} s^{2}+B_{1} s+C_{1}$. From Hurwitz Criterium, all roots of $p(s)$ have negative real parts, if, and only if, $A_{1}>0, B_{1}>0, C_{1}>0$ and $A_{1} B_{1}-C_{1}>0$. After a long computation one has

$$
A_{1}=\frac{2 R_{2}}{15 r_{2}}>0
$$

$$
\begin{gathered}
B_{1}=\frac{\left(\begin{array}{c}
16 \sqrt{R_{1} R_{2}} \Gamma\left(\frac{3 r_{2}}{2}\right)^{\frac{3}{2}} \\
\cdot\left(\begin{array}{c}
3 \gamma q_{1} r_{2}^{2}\left(9 r_{2}^{2}-4\right) \\
+25 q_{2}\left(1-r_{2}^{2}\right)
\end{array}\right) \\
+243 q_{1} q_{2}^{2}\left(1-r_{2}^{2}\right)^{2} r_{2}^{3} R_{2}^{2}
\end{array}\right)}{3600 q_{2}\left(1-r_{2}^{2}\right) r_{2}^{2} R_{2} \Gamma\left(\frac{3 r_{2}}{2}\right)}>0 \\
C_{1}=\frac{2 \sqrt{R_{1}} \sqrt{R_{2}} \sqrt{\Gamma\left(\frac{3 r_{2}}{2}\right)}}{135 r_{2}^{3}}>0
\end{gathered}
$$

$$
A_{1} B_{1}-C_{1}=\frac{\left(\begin{array}{l}
16 \gamma q_{1} \sqrt{R_{1} R_{2}} \Gamma\left(\frac{3 r_{2}}{2}\right)^{\frac{3}{2}} \\
\cdot\left(9 r_{2}^{2}-4\right) \\
+81 q_{1} q_{2}^{2}\left(1-r_{2}^{2}\right)^{2} r_{2} R_{2}^{2}
\end{array}\right)}{9000 q_{2}\left(1-r_{2}^{2}\right) r_{2} \Gamma\left(\frac{3 r_{2}}{2}\right)}>0
$$

Since is assumed that $\varepsilon>0$, it follows from (6), (8) that $q_{1}<0, q_{2}<0$, but this does not change the following conclusions. From (34) and (35) it follows that (39), (41) are true. And (40), (42) hold if, for example, $3 r_{2}-2 \geq$ 0 . This last condition is compatible, by (30), with the condition $r_{1}>1$. From this we have that (31) has an asymptotically stable equilibrium point. Thus, it follows from Averaging Theorem that (28) has an asymptotically stable periodic orbit near the point (32). Particularly, the first two coordinates of such orbit are near zero. And from (27) one gets that $x_{11}, u_{1}$ are near zero.

\section{QUENCHING}

In this section the body with mass $M$ in Figure 1 and Figure 4 will be referred as Main Body and the body with mass $m_{2}$ in Figure 4 as Secondary Body. 
From (18) and (15) one gets that the two first components of the stable periodic orbit of (14) obtained Section 3 can be written as

$$
\begin{aligned}
\bar{x}_{1}(\theta) & =\widehat{x}_{11}^{s} \cos (\theta)+\widehat{u}_{1}^{s}(\theta) \sin (\theta)+\mathrm{O}(\varepsilon) \\
\bar{u}(\theta) & =-\widehat{x}_{11}^{s} \sin (\theta)+\widehat{u}_{1}^{s} \cos (\theta)+\mathrm{O}(\varepsilon) .
\end{aligned}
$$

So, the amplitude of $\left(x_{1}(t), u(t)\right)$ of (12) is given by

$$
\sqrt{x_{1}^{2}+u^{2}}=\sqrt{\frac{2 \Gamma(1) q_{1}}{\lambda_{1} q_{2}}}+\mathrm{O}(\varepsilon) .
$$

This last result implies that, even for a periodic stable orbit, the Main Body of the non-ideal system given in Section 2 can suffer great oscillations in position and velocity when $\lambda_{1} \ll 1$, and this can be undesirable.

For the system given in Section 4 and assuming the stability conditions obtained in that section, one has from (32) that

$$
\begin{aligned}
z_{11}(\theta) & =\mathrm{O}(\varepsilon), \\
z_{1}(\theta) & =\mathrm{O}(\varepsilon), \\
z_{21}(\theta) & =\widehat{z}_{21}^{s}+\mathrm{O}(\varepsilon), \\
z_{2}(\theta) & =\widehat{z}_{2}^{s}+\mathrm{O}(\varepsilon), \\
\bar{p}_{1}(\theta) & =\widehat{p}_{1}^{s}+\mathrm{O}(\varepsilon) .
\end{aligned}
$$

Note that this means, in the coordinate system in which (28) is written, the Main Body is "almost" stopped. Anyway by using (45), (27), (24) and (26) one has the following estimate

$$
\sqrt{x_{1}^{2}+u^{2}} \leq \sqrt{\frac{16 R_{2} \Gamma\left(\frac{3 r_{2}}{2}\right)^{2}+R_{1}}{4 q_{2}^{2} R_{2}}}+\mathrm{O}(\varepsilon) .
$$

Comparing (46) with (44) and using (35) one has that the Main Body, in the mechanical system given in Section 4, has an uniformly bounded amplitude for all $\lambda_{1} \ll 1$. Thus the movement of the Main Body has been quenched due to the attachment of the Secondary Body.

\section{CONCLUSIONS}

In this paper, for the first time, a quenching result in a non-ideal system is rigorously obtained by using the Averaging Method. The main reason behind this result is a hypothesis on the angular momentum of the rotating parts of the energy source (motor) of the system. Estimates of the amplitude of the Main Body in the original system, see Figure 1 as well as the Main Body in the system given in the Section 4, Figure 4, are obtained proving the quenching.

The method used here can be applied in other interesting mechanical systems and it will be the subject of future research.

\section{ACKNOWLEDGMENTS}

The first author acknowledges the support given by Fundação de Amparo à Pesquisa do Estado de Minas GeraisFAPEMIG

\section{REFERENCES}

1. Balthazar, J.M., Mook, D.T., Brasil, R.M.L.R.F., Weber.H.I., Fenili, A., Belato, D. and Felix, J.L.P., 'Recent results on vibrating problems with limited power supply', in: Awrejcewicz, J., Brabski, J. and Nowakowski, J (eds), Sixth Conference on Dynamical Systems Theory and Applications, Lodz, Poland, December 10-12, 2001.

2. Balthazar, J.M., Mook, D.T., Brasil, R.M.L.R.F. Weber.H.I., Fenili, A., Belato, D. and Felix, J.L.P.,'An overview on Non-Ideal Vibrations', Meccanica 38, 2003, 613-621.

3. Balthazar, J.M., Brasil, R.M.L.R.F., Weber.H.I., Fenili, A., Belato, D., Felix, J.L.P. and Garzelli F.J. ' A Review of New Vibration Issues due to Non-Ideal Energy Sources', in Udwadia, F.E., Webwer, H.I. and Leitmann, G. (eds), Dynamical Systems and Control, Stability and Control: Theory, Methods and Applications 22, Chapman \& Hall/ CRC, Boca Raton, 2004.

4. Felix, J. L. P., Balthazar, J. M., Dantas, M. J. H. On energy pumping, synchronization and beat phenomenon in a nonideal structure coupled to an essentially nonlinear oscillator. Nonlinear Dyn. 56, 1-11 (2009).

5. Felix, J. L. P., Balthazar, J. M. , Dantas, M. J. H. On a Nonideal (MRD) Damper-Electro-Mechanical Absorber Dynamics. International Journal of Bifurcation and Chaos, Vol.21, No. 10, 2871âĂȘ2882, (2011).

6. Guckenheimer,J., Holmes, P. Nonlinear Oscillations, Dynamical Systems and Bifurcations of Vector Fields, Springer-Verlag, New York Inc. (1983).

7. Jiang, X. ,McFarland, D.M., Bergman, L.A., Vakakis, A.F. Steady state passive nonlinear energy pumping in coupled oscillators: theoretical and experimental results. Nonlinear Dyn. 33, 87-102 (2003).

8. Kononenko, V.O.Vibrating Systems with a Limited Power Supply Power, Iliffe Books Ltd (1969).

9. Malatkar,P., Nayfeh,A.H. Steady-state dynamics of a linear structure weakly coupled to an essentially nonlinear oscillator. Nonlinear Dyn. 47, 167-179 (2007).

10. Nayfeh, A.H. and Mook, D.T., Nonlinear Oscillations, John Wiley and Sons, New York, 1979.

11. Tondl, A., Quenching of Self-Excited Vibrations, Elsevier, Amsterdan, (1991) 
Copyright of AIP Conference Proceedings is the property of American Institute of Physics and its content may not be copied or emailed to multiple sites or posted to a listserv without the copyright holder's express written permission. However, users may print, download, or email articles for individual use. 